\title{
Research on Assistant System of Electric Power Distribution Planning Based on GIS
}

\author{
Dongqiang Heng ${ }^{\mathrm{a}}$, Xin Zhang ${ }^{\mathrm{b}}$, Shuzhe $\mathrm{Li}^{\mathrm{c}}$, Lei Zhang ${ }^{\mathrm{d}}$ \\ State Grid Henan Electric Power Company Luoyang Power Supply Company, \\ Luoyang 471000, China \\ a 296457845@qq.com, b1015903240@qq.com
}

Keywords: GIS, distribution network planning

\begin{abstract}
In this paper, with advanced computer technology and distribution network planning theory, studied with GIS-based distribution network planning aided design system. This paper analyzes the problems of distribution network planning, proposed the use of FME technology planning data integration and appreciation of thinking, to achieve a reasonable distribution network planning related GIS data and graphics data; secondly distribution network planning based on actual work demands, flexible data modeling methods to achieve the efficient management of data distribution network planning; Finally, according to the dynamic nature of the distribution network planning, multi-objective, non-linear, discrete, indeterminate features, the establishment of a spatial load analysis model and distribution network optimization model, and uses GIS path search algorithm auxiliary design features. The system for the power companies to achieve data integration planning and implementation of distribution network planning aided design has certain significance.
\end{abstract}

\section{Introduction}

With economic development, the growing social demand for electricity, power system stability has become a foundation of social stability. Power system stability requires not only a stable transmission system, also needs a stable power distribution system. Distribution system is the power system to the user's last link, the user is most closely connected, the impact on users supplies reliability, power quality and most direct. Domestic and international data show that $80 \%$ of the outage was caused due to the distribution system reliability; power loss of two-thirds is in the distribution system. Therefore, the distribution system safe, reliable and economical to run not only to the economic benefits of electric power companies, but also for improving customer satisfaction, reliability grid has a more important meaning. But our situation is a long time building power distribution system has not been given due attention, planning and research work is also focused on power system planning and power transmission system planning, while ignoring the study of distribution system planning, resulting in power distribution system lacks proper planning and construction, development is relatively backward. With the national "Eleventh Five-Year Plan" proposed energy efficiency goals to further clarify the distribution network planning requirements proposed update, for distribution network planning in China is facing great challenges.

Distribution planning have been in force since the grid has been in existence, it is accompanied by the development of new technologies, from the original hand drawings to CAD-assisted mapping plan, to today by means of geographic information systems implementation planning, network planning explanation has been considerable development. Domestic research on GIS-based distribution network planning aided design platform has made great progress, especially in theoretical research has had a lot of theoretical approaches can be effective row. In the context of the rapid development of the power system, distribution network planning for improving power quality, economy and security become increasingly important. In order to improve the distribution network planning of scientific, normative and efficient distribution network planning information become the development direction of the field. 


\section{Overview of Distribution Network Planning Aids System Based on GIS}

Distribution planning refers to a detailed analysis and research as well as the basis for future load status of urban distribution grid on the development of the power grid expansion and renovation plans, to meet the future capacity of users as possible, the reliability and power quality requirements of the situation next, so that the power company and the relevant departments under the process of obtaining better returns.

According to issue to divide the distribution system planning can be divided into spatial load forecasting, distribution network expansion planning, distribution automation planning and distribution of four parts reactive power planning:

Spatial load forecasting is the basis for the planning of the distribution system, is called spatial load forecasting it is because the distribution system planning, not only asked to predict the size of the load, but also required to predict the location of future load growth. Distribution network expansion planning is to meet the premise of the user supply and ensure various operational constraints and logical constraints, to find an optimal set of decision variables (new substation location, the installed capacity and construction time, the new feeder path, type and construction time, etc.) to implement network expansion and distribution system, and make the investment and operating costs of the minimum distribution network. Distribution automation plan, namely to achieve DA (Distribution Automation abbreviated as DA) planning functions in the distribution system. It includes SA (Substation Automation, referred to as SA) planning and FA (Feeder Automation, referred to as FA) planning. Distribution automation planning must matched with the distribution network expansion planning, or places the distribution network expansion planning. Reactive Power planning by the distribution network for reactive power compensation of distribution network voltage control and improve voltage quality and reduce network loss plan. Its main task is to identify new reactive power compensation equipment, the best location, capacity, type, and invest time.

GIS is a computer-based technology systems spatial data management and research. Surrounding the research, development and application of this technology is the formation of a cross-cutting edge of science. In computer software and hardware support, it can be spatial data by geographical coordinates or spatial location of various treatments, to study various spatial entities and their relations to the grid data graphical information provides a basis.

\section{Design of Distribution Network Planning System Based on GIS}

The system uses three-tier architecture. Data extraction layer using FME as a back-end data integration platform, and the distribution network planning data integration production MIS, GIS distribution systems, power marketing system, SCADA and other systems; build layer using Autodesk Map as the front-end GIS Development tools for building a data model for the distribution network planning implementation provides the basic platform; to achieve levels to accomplish the distribution network planning function show. Achieve specific functions layer implementation framework is the second development on Autodesk Map, the system keeps the basic common features of Autodesk Map, and add new features.

\subsection{Design of FME Data Interface}

FME system consists of three main engines: semantic transformation engine, it is FME's core module is responsible for the actual conversion work; semantic mapping file registry, which provides management of many different mechanisms for semantic mapping file, providing a simple filtering operation, which is used in the data conversion process to select the mapping file and registry provides classification mechanism for different users develop and share data conversion specification; automatic semantic mapping file generator, it enable FME to completely automatic way to perform the conversion. In this architecture, both semantic mapping file software from the registry or files generated from semantic mapping, semantic transformation engine are treated 
equally. So users do not develop in the mapping file mechanism, and it allows more focus on how to customize more complex tasks.

\subsection{Modeling Design of Distribution Network Planning}

The data model is a logical description of a part of the reality of the world. GIS data model to express the real world of geographic objects in digital form and their relationships. Data modeling is aimed at the computer abstract and express the real world, it will involve what specific data structure represents the real world, how to store real-world data.

The real world of houses, roads, power equipment is not isolated, but linked, this link performance when using GIS should be apparent, this is the data modeling process Relation of each object corresponds to a specific database is Contact between database tables. Between the drawing of buildings, roads, power facilities 3-7, between roads and buildings have a building belonging to a street relationship exists between the building and the building is that the electrical equipment electrical equipment power relationships, there is a relationship between a piece of electrical equipment belonging to the street between that position and other electrical equipment and roads. Data modeling is the process of the above-described real-world entities and relations into a computer describe objects and object relationships, and achieve the logical database design concepts. Autodesk's Autodesk Map 2006 provides a way to customize the built-OBJECT DATA establish GIS data object model; data model can be implemented in an external spatial database. Both methods have advantages and disadvantages, the first approach modeling, making full use of internal data storage mechanism provided by Autodesk, data query access speed is very fast; the use of an external database modeling spatial database is massive data store has a distinct advantage. In this paper, the first method is adopted; OBJECT DATA custom data objects include data objects defined, attached data objects to access data objects in three parts.

\section{Design of Spatial Load Analysis Model}

Spatial load analysis model can be divided into spatial situation analysis and spatial load forecasting model in two parts. The main task of spatial analysis model status of the load distribution system is the historical load data analysis, including analysis of nodes in the network load, terminal equipment load analysis. Load analysis is the basis of the grid with spatial load forecasting and distribution network planning. And the grid is different, in the distribution system, since the level of automation and other limitations, the distribution network load measuring less limited to a few large substations and transformer load user; and a large number of feeders and ordinary users Transformer no immediate measurement data, that is, in a sense, the distribution system is unobservable. To such an unobservable system load analysis, we must make full use of existing large amounts of electricity consumption data and user information, the establishment of appropriate load model, and through the load estimation and load forecasting method to compensate for the lack of actual measurement.

Spatial load forecasting is extended distribution network planning, distribution network automation planning and distribution of reactive power planning is fundamental to the distribution system is an important planning function modules. In the distribution planning system must be established spatial load forecasting model, using appropriate forecasting algorithm, to each cell in the region's future distribution network load forecasting, can the substation location, capacity, feeder type, path, switching equipment provided their input and time planning decision variables.

\section{Summary}

Along with the new energy-saving targets put forward, with the new demands for network planning, relying on past original hand drawings or CAD-assisted mapping planning approach has failed to meet the needs of their work, in order to better provide assistance for the distribution network planning decisions, proposed the establishment of a distribution network based on GIS platform aided design system planning goals. With the secondary distribution network planning platform, during the distribution plan, not only can quickly be digitized distribution network can 
also be used to provide data integration tools (FME), the original GIS data and planning data and reuse, solve the planning information "islands" and planning tools issues; the planning situation analysis provides a basis for network planning and transformation.

\section{Reference}

[1]Liu W, Zhang J, Liu N, et al. Design of a Distribution Network Intelligent Planning Platform Based on COTS[J]. Automation of Electric Power Systems, 2008.

[2]Zhang Y, Liu H, Jiang J, et al. Research on Evaluation Index and Method of Coordinated Planning Between Main System and Distribution Network[J]. Automation of Electric Power Systems, 2010, 34(15):37-41.

[3]Zhu Y, Yang J Y, Gao L J, et al. Research on optimal reactive power planning for distribution network containing asynchronous wind power generators[J]. Power System Protection \& Control, 2012.

[4]Wang, C., \& Wang, S. (2004). The intelligent planning of urban mid-voltage distribution network based on spatial gis part two automatic routing of multi-sectioned and multi-linked network. Automation of Electric Power Systems, 28(6), 55-59.

[5]Liu J, Liu Z, Huang W, et al. An Intelligent Distribution Network Planning Method Based on Geographical Differential Evolution[J]. Automation of Electric Power Systems, 2007, 31(2):32-35.

[6]Lin H, Wen-Xuan W U, Han W U, et al. Optimal Segmentation of Power Supply Model of MV Distribution System Based on SLCC[J]. East China Electric Power, 2013. 